\title{
miR-34a regulates adipogenesis in porcine intramuscular adipocytes by targeting ACSL4
}

\author{
Wenwen Wang ${ }^{\dagger}$, Xiuxiu Li ${ }^{\dagger}$, Ning Ding, Jun Teng, Shen Zhang, Qin Zhang and Hui Tang ${ }^{*}$ (D
}

\begin{abstract}
Background: Intramuscular fat (IMF) content is an important factor in porcine meat quality. Previously, we showed that miR-34a was less abundant in liver tissue from pigs with higher backfat thickness, compared to pigs with lower backfat thickness. The purpose of this present study was to explore the role of miR-34a in adipogenesis.

Result: Bioinformatics analysis identified Acyl-CoA synthetase long chain family member 4 (ACSL4) as a putative target of miR-34a. Using a luciferase reporter assay, we verified that miR-34a binds the ACSL4 mRNA at the 3'UTR. To examine the role of the miR-34a-ACSL4 interaction in IMF deposition in the pig, mRNA and protein expression of the ACSL4 gene was measured in primary intramuscular preadipocytes transfected with miR-34a mimic and inhibitor. Our results showed that ACSL4 is expressed throughout the entire differentiation process in pig preadipocytes, similar to the lipogenesis-associated genes PPARY and aP2. Transfection with miR-34a mimic reduced lipid droplet formation during adipogenesis, while miR-34a inhibitor increased lipid droplet accumulation. Transfection with miR-34a mimic also reduced the mRNA and protein expression of ACSL4 and lipogenesis genes, including PPARY, aP2, and SREBP-1C, but increased the expression of steatolysis genes such as ATGL and Sirt1. In contrast, the miR-34a inhibitor had the opposite effect on gene expression. Further, knockdown of ACSL4 decreased lipid droplet accumulation.
\end{abstract}

Conclusions: Our results support the hypothesis that miR-34a regulates intramuscular fat deposition in porcine adipocytes by targeting ACSL4.

Keywords: IMF, miR-34a, ACSL4, Pig

\section{Background}

Intramuscular fat (IMF) content is a primary indicator of porcine meat quality [1]. An increase in IMF content can improve meat flavor [2]. However, substantial efforts have been made to improve production efficiency and select for lean growth, both of which impact IMF negatively. Selection for enhanced IMF has therefore become an important focus in pork production.

\footnotetext{
* Correspondence: tanghui@sdau.edu.cn

tWenwen Wang and Xiuxiu Li contributed equally to this work.

Shandong Provincial Key Laboratory of Animal Biotechnology and Disease Control and Prevention, Shandong Agricultural University, No. 61, Daizong

Street, Tai'an City 271018, Shandong Province, China
}

High-throughput methods, such as genome-wide association studies and transcriptome expression profiling, have been used to search for genes that potentially affect fat deposition in swine. Many genes associated with an extreme capacity for IMF deposition have been identified [3-9]. In addition to the multiple genes that influence adipogenesis, IMF is also likely to be under the control of posttranscriptional regulatory factors such as microRNAs. MicroRNAs are small non-coding RNA molecules that regulate gene expression by targeting mRNA transcripts for cleavage or translational inhibition [10,11]. miRNAs play important roles in various biological processes, including cell differentiation, proliferation and apoptosis [12], organ development [13], lipid metabolism [14], and

(c) The Author(s). 2020 Open Access This article is licensed under a Creative Commons Attribution 4.0 International License, which permits use, sharing, adaptation, distribution and reproduction in any medium or format, as long as you give appropriate credit to the original author(s) and the source, provide a link to the Creative Commons licence, and indicate if changes were made. The images or other third party material in this article are included in the article's Creative Commons licence, unless indicated otherwise in a credit line to the material. If material is not included in the article's Creative Commons licence and your intended use is not permitted by statutory regulation or exceeds the permitted use, you will need to obtain permission directly from the copyright holder. To view a copy of this licence, visit http://creativecommons.org/licenses/by/4.0/ The Creative Commons Public Domain Dedication waiver (http://creativecommons.org/publicdomain/zero/1.0/) applies to the data made available in this article, unless otherwise stated in a credit line to the data. 
tumorigenesis [15]. Emerging evidence suggests that miRNAs are also involved in adipogenesis. For example, miR196a induces preadipocyte differentiation by increasing adipocyte marker expression, lipid accumulation, and triglyceride content [16]. miR-27a-5p increases fat deposition in steers partly by targeting the calcium-sensing receptor (CASR) [17]. miR-30e regulates adipocyte differentiation by targeting the low-density lipoprotein receptor-related protein 6 [18]. miR-155, miR-130, and miR-210 inhibit adipocyte formation by targeting the key adipogenic transcriptional factors PPAR , C/EBP $\alpha$, and TCF712 in the $\mathrm{Wnt} / \beta$-catenin signaling pathway $[19,20]$. We previously identified potential miRNAs regulators of porcine fat deposition by using high-throughput sequencing to examine the transcriptomes in animals with extreme differences in backfat thickness. One of the miRNAs, miR-34a, is markedly less abundant in animals with higher backfat thickness (H group) compared with those with lower backfat thickness (L group) [9]. This result suggests that miR-34a may play important roles in porcine adipogenesis.

MicroRNA-34a has attracted interest recently because of its ability to modulate a myriad of oncogenic functions in different cancers [21-27]. Not only does it play a role in cancer metastasis $[28,29]$ and drug resistance [30], it is now being evaluated as a diagnostic as well as a prognostic biomarker [31-33]. In addition, a miR-34a inhibitor has been identified that may effectively protect against sevoflurane-induced hippocampal apoptosis by targeting Wnt1 and activating the Wnt/B-catenin pathway [34]. miR-34a is involved in the pathogenesis of non-alcoholic fatty liver disease [35] and is down-regulated in genetically improved farmed tilapia (Oreochromis niloticus) when they are fed a high-fat diet [36]. However, little is known about the role of miR-34a in porcine adipogenesis.

To explore the function of miR-34a in swine, we used bioinformatics analyses to predict its interactions, and conducted experiments to test our predictions using primary preadipocytes. The results provide insight into the ways in which non-coding RNAs affect IMF in pigs.

\section{Results}

\section{Biological functions of miR-34a based on target analysis}

To explore the possible biological functions of miR-34a, the TargetScan and miRDB algorithms were used to predict miR-34a targets. Seven hundred fifty-four and five hundred forty-seven targets were predicted with TargetScan and miRDB, respectively (Supplementary Tables S1 and S2). Two hundred ninety-eight genes overlapped with the targets (Supplementary Table S3), and were examined for potential biological roles using Gene Ontology (GO) term enrichment and Kyoto Encyclopaedia of Genes and Genomes (KEGG) pathway analyses. Several molecular function categories were enriched in the GO analysis (Fig. 1a). Fatty acid biosynthesis and the phosphatidylinositol signaling system were significantly enriched in the KEGG analysis (Fig. 1b).

The mature miR-34a sequence is highly conserved in various species, including human, mouse, and rat (Fig. 2a). miRNA target prediction identified ACSL4 as a potential target gene of miR-34a, with an estimated free energy of $-29.2 \mathrm{kcal} / \mathrm{mol}$ for the interaction between them. ACSL4 encodes acyl-CoA synthetase long chain family member 4 , which generates fatty acyl-CoA esters from long-chain fatty acids. The putative target site in the ACSL4 mRNA is shown in Fig. 2b.

\section{Interaction between miR-34a and ACSL4}

To verify that ACSL4 is a target of miR-34a, we tested their ability to interact in $293 \mathrm{~T}$ cells using a dualluciferase reporter system. miR-34a mimic significantly decreased luciferase activity generated by the wild-type $A C S L 4$ reporter vector, compared to the negative control $(P<0.01)$. In contrast, luciferase activity was not affected when a mutated version of the putative miR-34a interaction region was transfected into the cells (Fig. 3a). These results support the hypothesis that ACSL4 mRNA is targeted by miR-34a. We further detected the expression of ACSL4 in muscle tissue, which revealed a higher expression in the $\mathrm{H}$ group than that in the L group (Fig. $3 \mathrm{~b}$ ).

\section{Expression of $A C S L 4$ during porcine preadipocyte differentiation}

To examine whether ACSL4 is a potential contributor to IMF deposition, expression of ACSL4 was measured by qRT-PCR during preadipocyte differentiation $(0,2,4,6$, and 8 days after induction). Other marker genes that are widely used in studies of lipid metabolism [37] such as PPARY, aP2, ATGL, and Sirt1 were also included. As shown in Fig. 4, expression of ACSL4 mRNA gradually increased after adipocytes were induced to differentiate. Expression peaked at 4 days, the time at which a majority of preadipocytes differentiated into mature adipocytes, and then declined steadily (Fig. 4). Interestingly, similar expression patterns were also observed for lipogenesis transcripts such as PPARy and $a P 2$ (Fig. 4). In contrast, expression of the steatolysis genes $A T G L$ and Sirt1 increased steadily during preadipocyte differentiation (Fig. 4). The results are consistent with the hypothesis that ACSL4 is involved in lipogenesis.

\section{miR-34a inhibits lipogenesis by targeting ACSL4}

The results of the dual luciferase assay described earlier strongly suggested that miR-34a and ACSL4 mRNA interact. To test if miR-34a affects lipid metabolism, a mimic and an inhibitor of miR-34a were transfected into porcine preadipocytes. As shown in Fig. 5a, the miR-34a mimic was detected after transfection, with the highest 


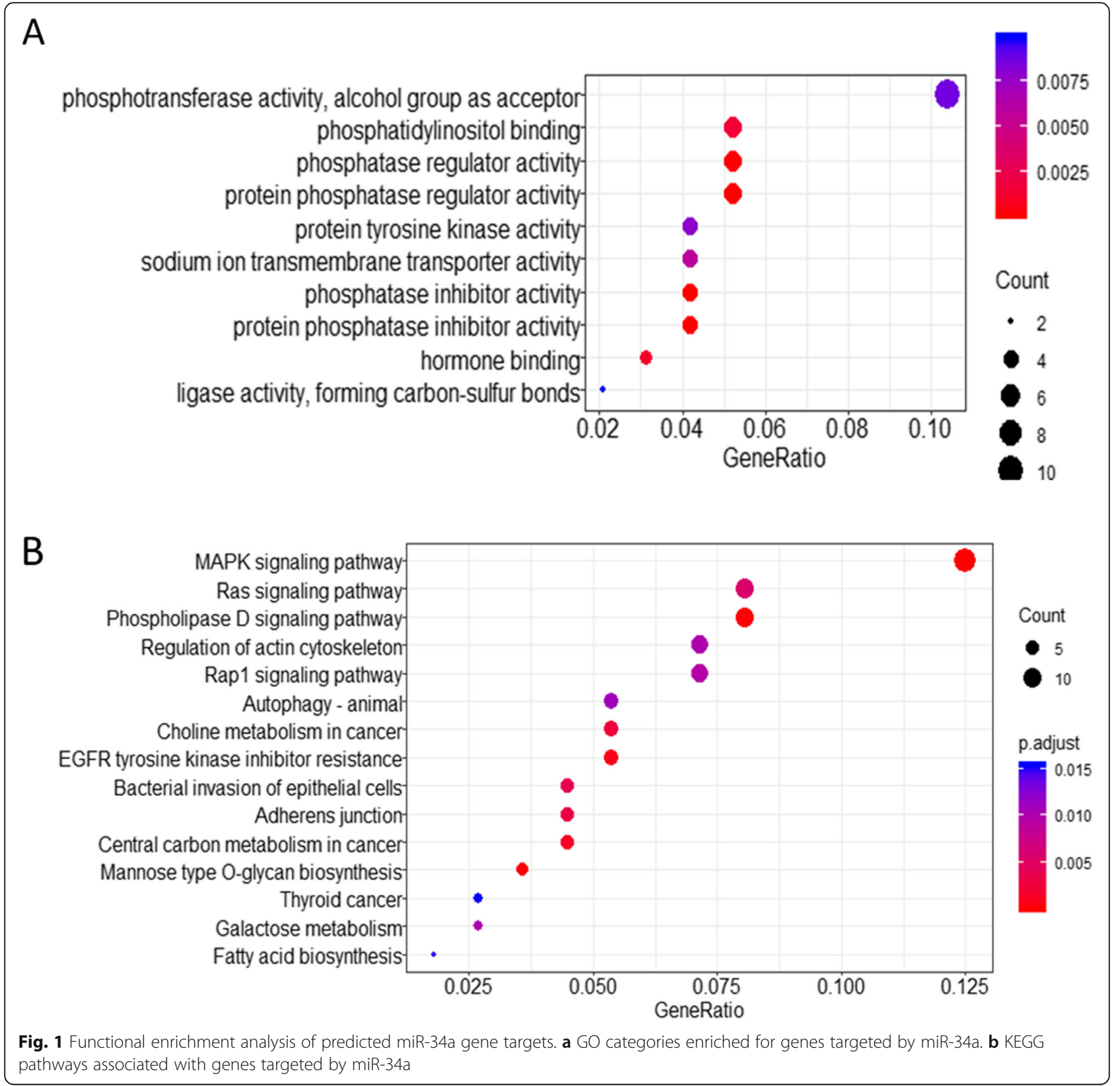

levels observed after $48 \mathrm{~h}$. We then used qRT-PCR and western blotting to measure mRNA and protein expression of ACSL4 and other genes related to lipid metabolism $48 \mathrm{~h}$ after transfection of preadipocytes with miR34a mimic and inhibitor (Fig. 5b, c, d). As expected, transfection with miR-34a mimic significantly suppressed mRNA and protein expression of ACSL4 and other lipogenesis genes, including PPAR,$a P 2$, and SREBP-1C, and increased expression of steatolysis genes, such as $A T G L$ and Sirt1. In contrast, miR-34a inhibitor had the opposite effect on the mRNA and protein expression of lipogenesis and steatolysis-related genes, suggesting that miR-34a inhibits lipogenesis by targeting
ACSL4. Consistent with this result, Oil Red O and triglyceride (TG) quantification assays revealed that the miR34a mimic significantly decreased lipid droplet numbers, while the miR-34a inhibitor increased them (Fig. 5e, f).

\section{Silencing of the ACSL4 gene decreases accumulation of} lipid droplets

RNA interference was used to investigate the function of ACSL4 in adipogenesis. Three si-ACSL4 fragments (si1ACSL4, si2-ACSL4, si3-ACSL4) were designed (Supplementary Table S4), and si3-ACSL4 was found to have the highest interference effect (Fig. 6a, b, c). Further, Oil Red $\mathrm{O}$ and $\mathrm{TG}$ quantification assays showed that 


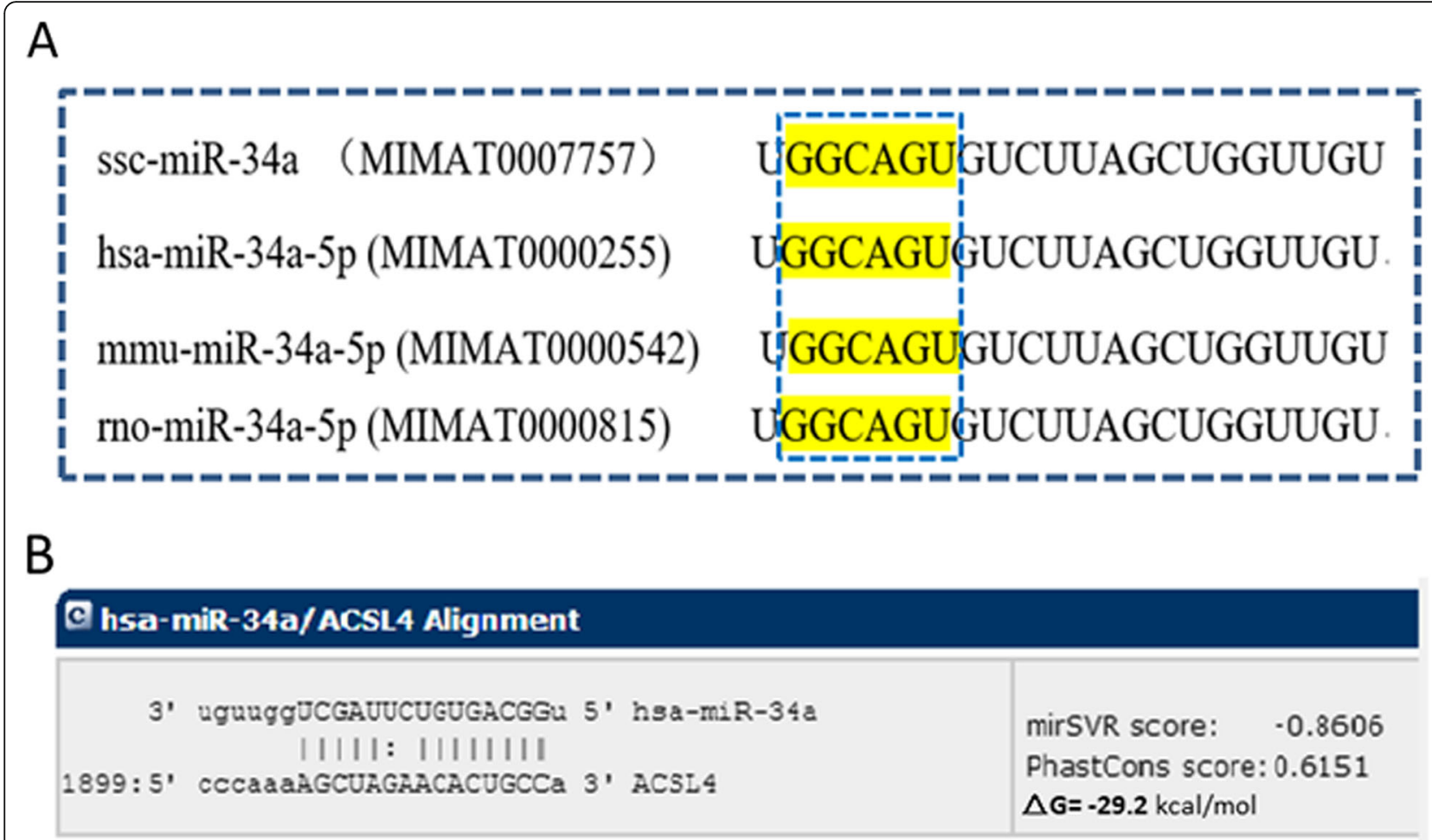

Fig. 2 Bioinformatics analysis of miR-34a. a Mature sequence of miR-34a is conserved among species including swine (ssc), human (hsa), mouse $(\mathrm{mmu})$, and rat (rno). Data were obtained from miRBase (www.mirbase.org/). b Predicted interaction between ACSL4 3'UTR and miR-34a
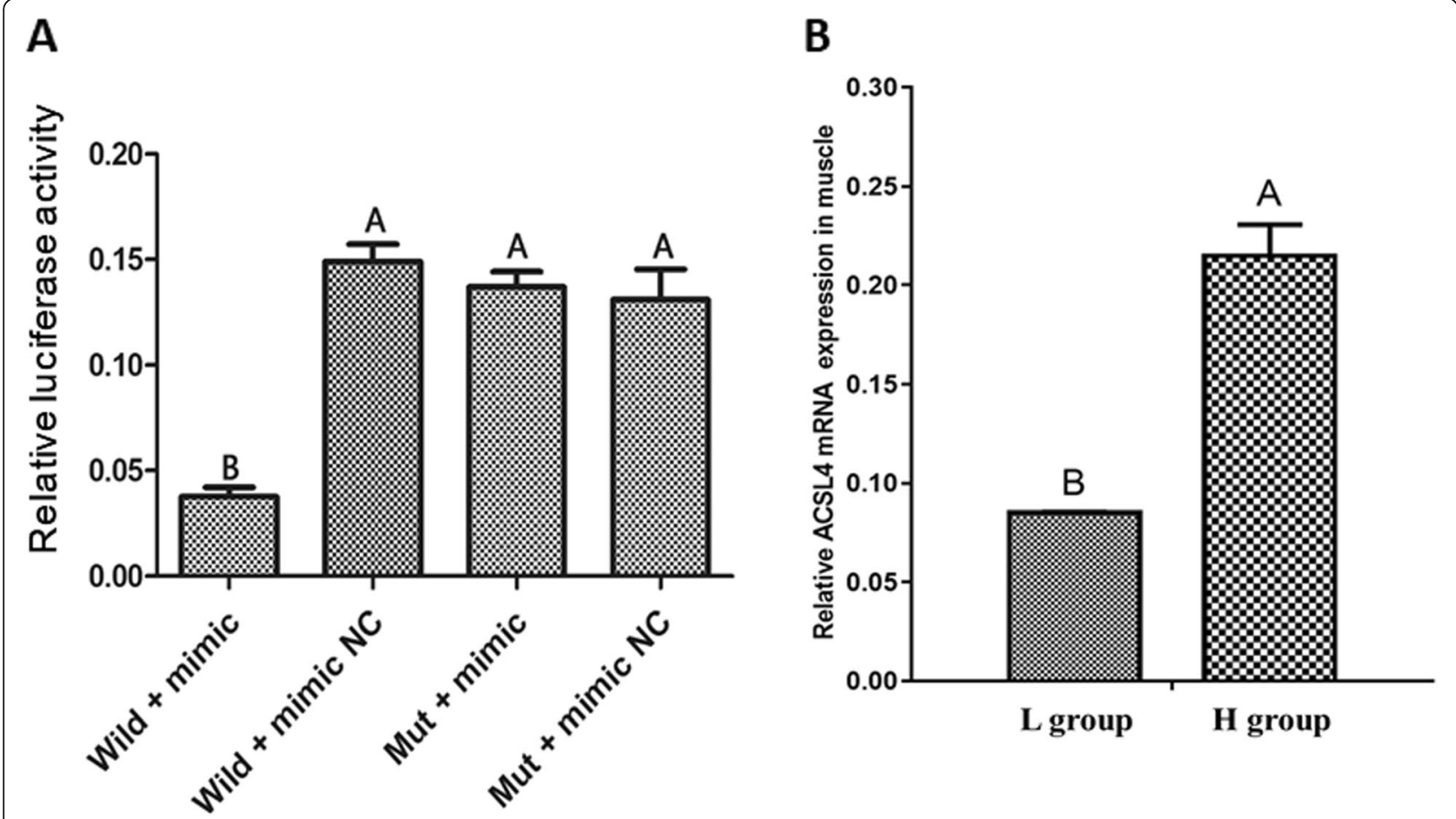

Fig. 3 a Dual luciferase reporter assay to detect targeting of ACSL4 by miR-34a in 293 T cells. $\mathbf{b}$ The relative expression of ACSL4 mRNA in muscle tissues obtained from animals in the $H$ and $L$ groups. Results are presented as means \pm SE of three independent determinations. Labels (A vs. B) indicate significantly different values $(P<0.01)$ 


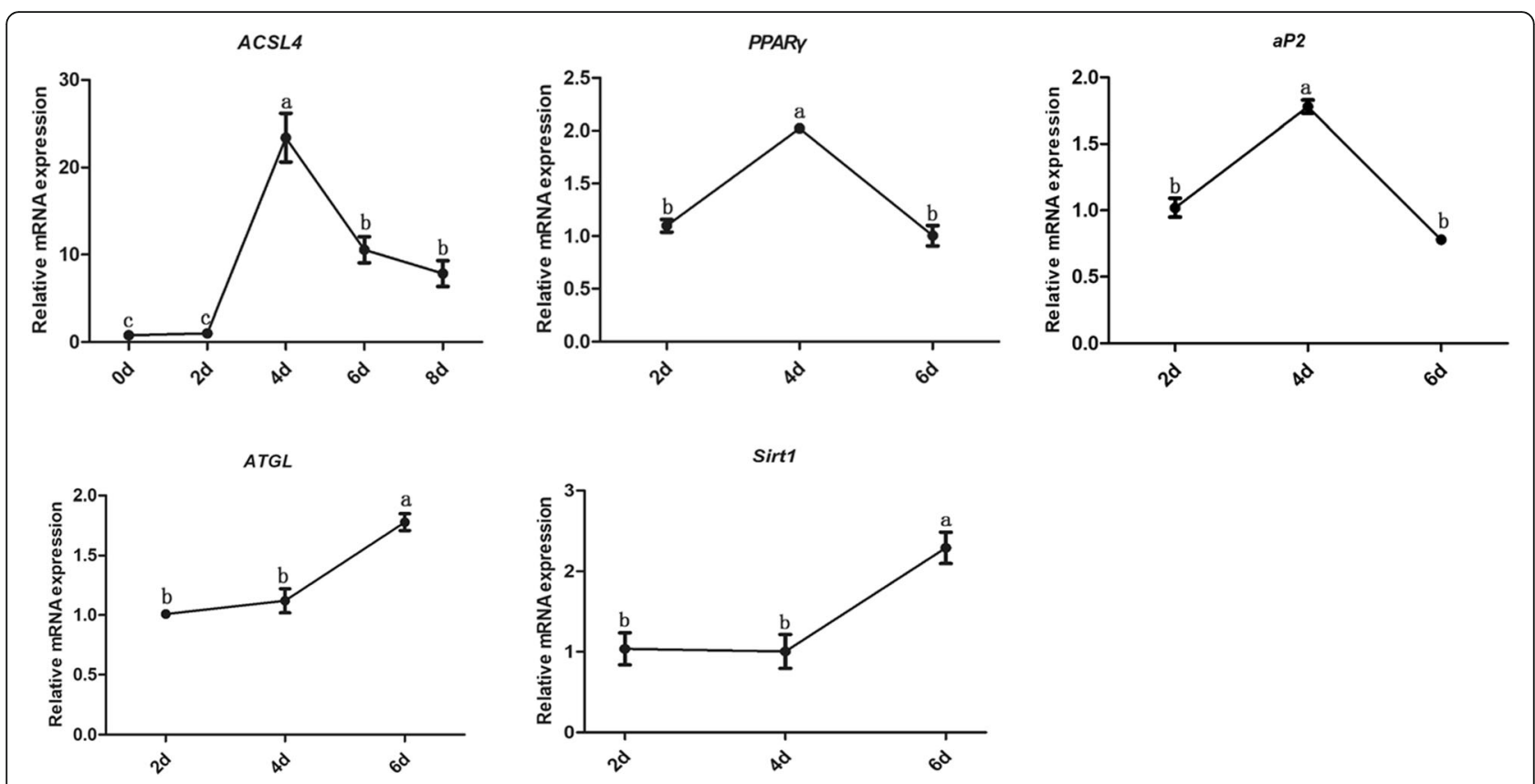

Fig. 4 Expression of ACSL4 and other lipid metabolism-associated genes during preadipocyte differentiation in vitro $(0,2,4,6$, and 8 days). The results are presented as means \pm SE of three independent determinations. Labels $(a, b, c)$ indicate significantly different values $(P<0.05)$

knockdown of ACSL4 reduced lipid droplet accumulation (Fig. 6d, e), similar to the effect of miR-34a mimic (Fig. 5e, f). Taken together, these results demonstrated that miR-34a negatively regulates adipogenesis in porcine adipocytes by targeting ACSL4.

\section{Discussion}

Numerous miRNAs regulate adipogenesis by interacting with transcription factors or important signaling molecules that are involved in adipocyte differentiation [38]. Based on our previous studies, miR-34a is less abundant in liver tissue from pigs with higher backfat thickness, compared with pigs with lower backfat thickness [9]. Bioinformatics analysis suggested that potential miR-34a targets functioned in MAPK signaling, regulation of the actin cytoskeleton, galactose metabolism, and fatty acid biosynthesis (Fig. 1). The potential involvement of miR-34a in fatty acid biosynthesis, in combination with the high expression of miR-34a in pigs with low backfat thickness, led us to hypothesize that miR-34a functions in lipid metabolism.

In this study, we investigated the mechanism by which miR-34a affects lipid metabolism in pigs. Sequence analysis suggested that miR-34a targets the ACSL4 mRNA within the 3'UTR (Fig. 2). In a dual luciferase assay, overexpression of miR-34a inhibited luciferase activity generated by wild-type ACSL4, but did not affect activity of a construct containing a mutated version of the putative miR-34a interaction region (Fig. 3a). The porcine ACSL4 gene is a member of the long-chain acyl-CoA synthetase (ACSL) family of enzymes that catalyze the addition of a coenzyme-A (CoA) group to a fatty acid to form fatty acyl-CoAs $[39,40]$. Five ACSL isoforms can each activate and channel various fatty acids to different metabolic fates [40]. Proposed functions of ACSL4 include intracellular lipid storage [41], cholesterol transport from the endoplasmic reticulum into the mitochondria [42], and regulation of arachidonic acid and its metabolites [43-46]. In addition, ACSL4 polymorphism is associated with IMF content and fatty acid composition in different pig breeds [47-49].

To verify that ACSL4 plays a role in the regulation of lipid metabolism, we measured the expression of ACSL4 during porcine preadipocyte differentiation. ACSL4 mRNA was expressed throughout the entire differentiation process, and abundant in middle term after adipocyte differentiation (Fig. 4). By studying adipocyte differentiation in vitro using various preadipose cell lines and primary preadipocytes, it has been possible to dissect the molecular and cellular events that occur during the transition from undifferentiated fibroblast-like preadipocytes into mature round fat cells [50]. As with primary human, mouse, and rat preadipocyte cell lines, primary pig preadipocytes also proliferate and differentiate, becoming adipocytes with lipid droplets in vitro (Supplementary Figure S1) [51]. We also analyzed the expression of PPAR $y$ and $a P 2$, which are prominent adipocyte marker genes, and $A T G L$ lipases and deacetylase Sirt1 [52]. Similar expression patterns were observed for ACSL4 and lipogenesis-associated genes, while the expression patterns for the steatolysis genes were different (Fig. 4). Also, the expression of ACSL4 mRNA in muscle 

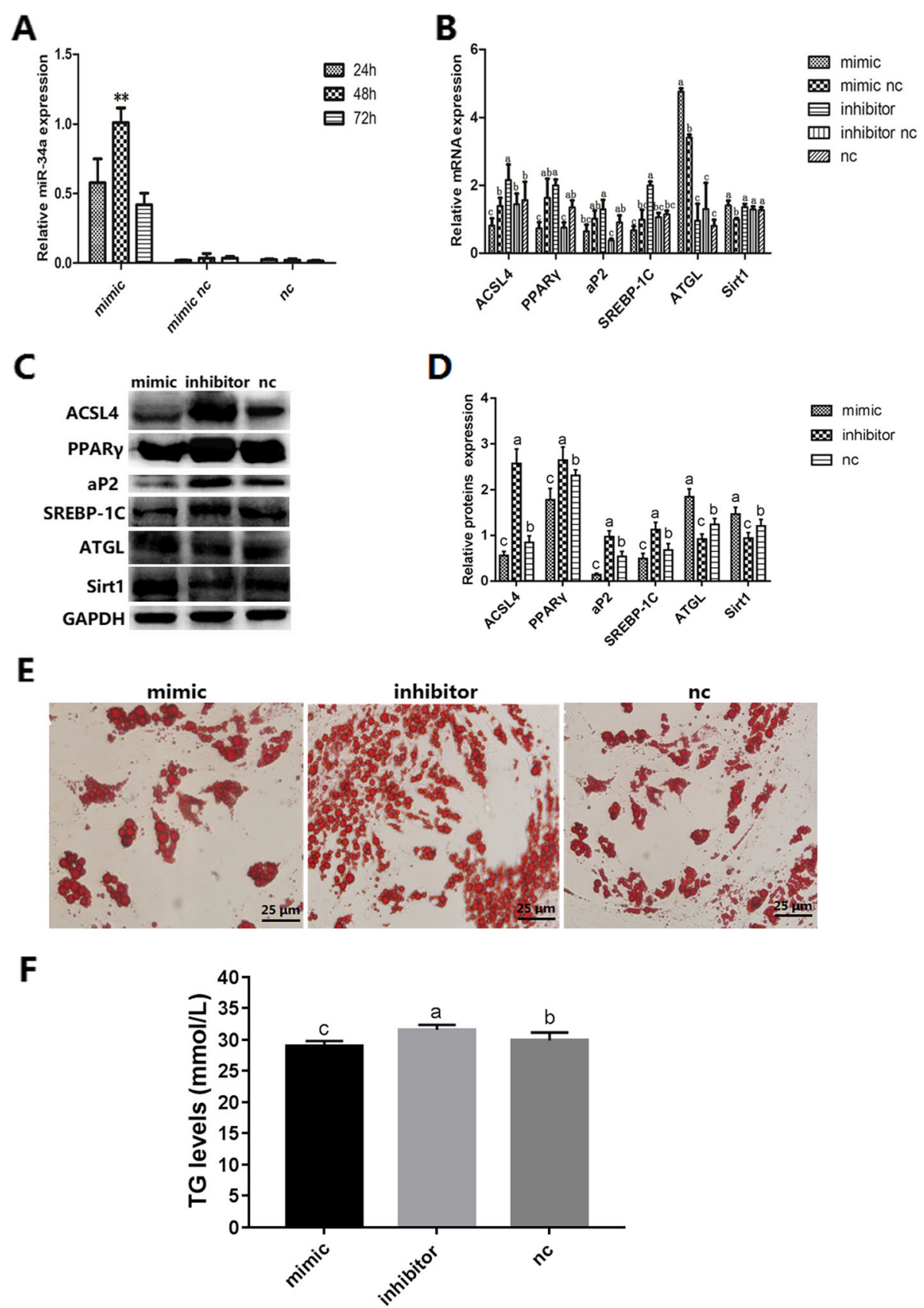

Fig. 5 miR-34a inhibits lipogenesis in porcine preadpocytes. a Detection of miR-34a mimic after transfection for 24, 48 , and $72 \mathrm{~h}$. mimic nc: mimic negative control; nc: blank negative control. $\mathbf{b}$ Expression of mRNA from genes related to lipogenesis and steatolysis in differentiated cells transfected with miR-34a mimic, mimic negative control, inhibitor, inhibitor negative control, and blank negative control. c Proteins detected by western blotting in the mimic, inhibitor, and nc groups. $\mathbf{d}$ Protein expression in the mimic, inhibitor, and nc groups. e Oil Red O staining of terminally differentiated adipocytes. $\mathbf{f}$ TG levels in the mimic, inhibitor, and nc groups. Results are presented as means $\pm \mathrm{SE} ; n=3 ;{ }^{*} P<0.01$. Labels $(a, b, c)$ indicate significantly different values $(P<0.05)$

tissue is higher in the $\mathrm{H}$ group than that in the $\mathrm{L}$ group (Fig. 3b). This suggests that ACSL4 is involved in pig lipogenesis.

To further investigate whether interaction between miR-34a and ACSL4 mRNA plays a role in lipid deposition, miR-34a mimic, inhibitor, and NC were transfected into porcine preadipocytes. As expected, miR-34a mimic decreased the number of lipid droplets, resulting in lower lipid content levels compared to cells transfected with NC. In contrast, miR-34a inhibitor increased the lipid droplet number, resulting in higher lipid content levels (Fig. 5e, f). Our results are consistent 


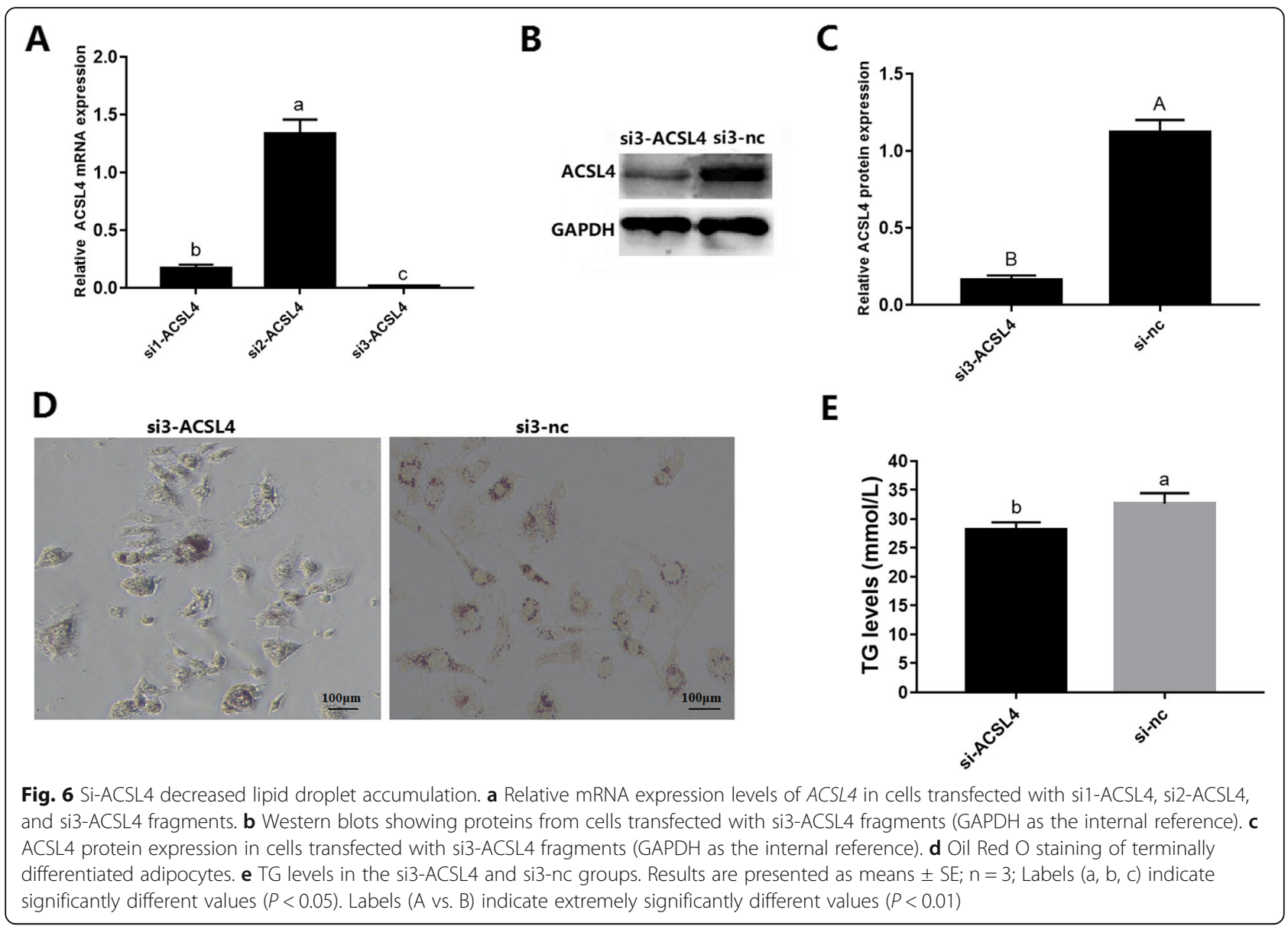

with one previous study [53], but are inconsistent with a recent report that overexpression of miR-34a increases lipid deposition in mouse liver and HepG2 cells [54]. The discrepancy may be due to differences between species or cell type. A particular miRNA may play different roles at different developmental stages within one cell type, or at the same developmental stage in different cell types [16]. We also investigated whether miR-34a regulates the expression of lipogenesis- and steatolysisrelated genes. As shown in Fig. 5, miRNA-34a mimic decreased the expression of ACSL4 and lipogenesis genes (PPARY, aP2 and SREBP-1C) and increased expression of steatolysis genes (ATGL and Sirt1). In contrast, the miR-34a inhibitor had the opposite effect on gene expression. Using RNA interference, we investigated the function of ACSL4 in adipogenesis. The results showed that knockdown of ACSL4 reduced lipid droplet accumulation (Fig. 6d, e), similar to the effect of miR-34a mimic (Fig. 5e, f). Taken together, the results demonstrate that miR-34a negatively regulates adipogenesis in porcine adipocytes by targeting ACSL4. A checklist for microRNA-target interactions (MTI) is presented in Table 1, following the recommended standards for an MTI report [55].

\section{Conclusions}

In this study, we investigated the mechanism by which miR-34a affects lipid metabolism in pigs. First, we demonstrated that miR-34a binds the ACSL4 mRNA at the 3'UTR using a luciferase reporter assay. We then showed that transfection of porcine preadipocytes with miR-34a mimic reduced lipid droplet formation, while transfection with miR-34a inhibitor increased the accumulation of lipid droplets. Further, knockdown of ACSL4 also decreased lipid droplet accumulation. Together, our data support the conclusion that miR-34a negatively regulates lipogenesis in porcine adipocytes by targeting ACSL4.

\section{Methods}

miRNA target gene prediction and functional analyses miRNA targets were predicted using TargetScan 7.2 (http:// www.targetscan.org/) [56] and miRDB (http://mirdb.org) [57]. The free energy of the miR-34a-ACSL4 interaction was calculated using RNAhybrid 2.2 (https://bibiserv.cebitec.uni-bielefeld.de/rnahybrid) [58]. GO term and KEGG enrichment analyses for overlapping target genes were performed using the $\mathrm{R}$ package 'clusterProfiler' [59], with $p$-values calculated using right-sided hypergeometric tests. Figures were prepared using the R package 'ggplot2' [60]. 
Table 1 Checklist for experimentally validated miRNA-target interaction (MTI)

\begin{tabular}{ll}
\hline Item & Results \\
\hline 1. miRNA gene name (Entrez ID) & miR-34a (100316602) \\
2. Target gene name (Entrez ID) & ACSL4 (448980) \\
3. Species name (Species ID) & Sus scrofa domesticus (9825) \\
4. Genomic location of MTI Nucleotide sequence & $5^{\prime} C A C T G C C$ \\
Location according to Ensembl & X:89761656-89761650 \\
Location within a part of a gene & 1934-1940 (location within 3'UTR) \\
Method for experimental validation & Luciferase reporter assay, qPCR, western blot \\
Cell lines & 293 T cell lines, porcine primary intramuscular preadipocytes \\
5. Sequence variants within MTI & None \\
6. Associated phenotype & Adipogenesis
\end{tabular}

Note:Checklist was prepared according to guidelines for the miRNA target reporting standardization [55]

\section{Dual luciferase reporter assay}

The mature miR-34a sequence was retrieved from miRBase (http://www.mirbase.org/). The ACSL4 3'UTR wild type (WT) and mutated (MUT) sequences were cloned into pmirGLO vectors using the SacI and SalI restriction sites. Primers for the luciferase reporter assay are listed in Supplementary Table S5. Two hundred ninety-three T cells in logarithmic growth phase were seeded into the wells of a 96-well plate. Upon reaching $80 \%$ confluence, the cells were co-transfected with ACSL4-3'UTR-Wild plasmid and miR-34a mimic using Lipofectamine 2000. ACSL4-3'UTR-Wild + miR-34a mimic NC, ACSL43'UTR-Mut + miR-34a mimic, and ACSL4-3'UTR-Mut + miR-34a mimic NC were also transfected for comparison. After $48 \mathrm{~h}$, luciferase activity was determined with the dual-luciferase reporter assay system (Promega, USA). All luciferase assays were performed in triplicate and the experiment was performed three times.

\section{Isolation, culture, differentiation, and transfection of porcine primary intramuscular preadipocytes}

Six 7-day old Yorkshire were purchased from the experimental farm at the Chinese of Academy Agricultural Sciences. Animals were humanely euthanized by electrical stunning. The longissimus dorsi muscle (LD) was removed from piglets under sterile conditions. Visible connective tissue was removed, and the remaining tissue was finely minced. Following the protocol described in a previous study [53], preadipocytes were isolated using differential rate adherence by subjecting the tissues to digestion for $2 \mathrm{~h}$ with $0.1 \%$ type II collagenase. The digested sample was filtered aseptically through a $200 \mu \mathrm{m}$ nylon mesh filter to isolate cells. The filtered and washed cells were seeded at a density of $2.5 \times 10^{5}$ cells per $35-\mathrm{mm}$ culture dish in DMEM/F12 medium with $10 \%$ fetal bovine serum (Hyclone, Logan, UT, USA), supplemented with penicillin (100 U/ $\mathrm{mL})$ and streptomycin $(100 \mathrm{U} / \mathrm{mL})$. The cells were incubated at $37^{\circ} \mathrm{C}$ under a water-saturated atmosphere containing $95 \%$ air and $5 \% \mathrm{CO}_{2}$. After $2 \mathrm{~h}$, the dishes were washed with a PBS solution 2-3 times to remove nonadherent cells and to obtain the precursor intramuscular-muscle fat cells. Subsequently, fresh complete culture solution was added and replaced every 2 days. After 2 days, the majority of cells had adhered to the culture dish walls (Supplementary Figure S1A). The number of adherent cells continued to increase with time, and spreading cells had triangular or fusiform shapes. After 8 days, cells had formed a single layer and were morphologically similar to primary cells (Supplementary Figure S1B). To examine IMF deposition and droplet morphology in cultured intramuscular adipocytes, cells were collected 2, 4, 6, and 8 days after induction of differentiation and then stained using Oil Red O. After 2 days of induction, a small number of lipid droplets were detected (Supplementary Figure $\mathrm{S} 1 \mathrm{C})$. The abundance of lipid droplets increased gradually from 4 to 6 days (Supplementary Figure S1D, E), and a large number of lipid droplets were apparent at 8 days (Supplementary Figure S1F). The results showed that the isolated cells were intramuscular preadipocytes.

When the cells reached $80 \%$ confluence, they were divided into two groups. The first group was induced to differentiate from preadipocytes to adipocytes. The differentiation medium (AIM; adipocyte-inducing medium) consisted of base medium supplemented with $0.5 \mathrm{mM}$ isobutyl methylxanthine (IBMX, Sigma-Aldrich, St. Louis, MO, USA), 1.0 $\mu$ M dexamethasone (DEX, Sigma, USA) and $1.0 \mu \mathrm{g} / \mathrm{mL}$ insulin (INS, Sigma). After addition of differentiation medium, cells were collected at $0,2,4$, 6 , and 8 days. The second group was used for overexpression and knockdown experiments. These cells were starved for $12 \mathrm{~h}$ in Opti-MEM (Gibco, USA), and then transfected with miR-34a mimic (artificially synthesized miR-34a mimic, $100 \mathrm{nM}$ ), mimic negative control (NC, $100 \mathrm{nM}$ ), inhibitor (anti-miR-34a, $100 \mathrm{nM}$ ), inhibitor NC $(100 \mathrm{nM})$ and si1-ACSL4 (100 nM), si2-ACSL4 (100 
nM), si3-ACSL4 (100 nM). ACSL4 siRNA sequences were designed by Shenggong technology Co., LTD. All sequences are listed in Supplementary Table S4. Transfection was conducted with Lipofectamine 2000 (Invitrogen, USA) according to manufacturer's protocol. Transfected preadipocytes were cultured in 6-well plates for 2 days in the presence of AIM, followed by treatment with insulin alone for 1 more day.

\section{Oil red $O$ staining}

Cells were washed three times with phosphate-buffered saline (PBS) and then fixed in $4 \%$ paraformaldehyde for $30 \mathrm{~min}$. The cells were washed twice with deionized water and stained with $60 \%$ Oil Red O (solvent: isopropanol, $0.5 \mathrm{~g}$ Oil Red powder $/ 100 \mathrm{~mL}$ ). The cells were protected from light for $30 \mathrm{~min}$, washed three times with PBS, and examined under a microscope.

\section{Determination of triglyceride}

TG levels were quantified using a GPO-PAP enzyme assay (Jiancheng, China). The cells were digested with achromatic trypsin and centrifuged for $10 \mathrm{~min}$ at a speed of $1000 \mathrm{rpm}$. The pellet was washed twice with PBS, and the cells were resuspended at $10^{6} / \mathrm{mL}$ with ultrapure water. The cells were disrupted by sonication $(3-5 \mathrm{~s}$ bursts, 3-5 repetitions) or manually while being chilled in an ice bath. The cells were transferred into 96 well plates and divided into three groups. The blank group

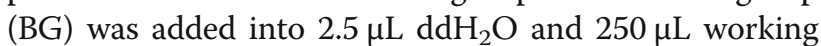
fluid. The standard group (SG) was added into $2.5 \mu \mathrm{L}$ TG $(2.26 \mathrm{mmol} / \mathrm{L})$ and $250 \mu \mathrm{L}$ working fluid. The experimental group (EG) was added into $2.5 \mu \mathrm{L}$ cells and $250 \mu \mathrm{L}$ working fluid. The reactions were mixed and incubated at $37^{\circ} \mathrm{C}$ for $10 \mathrm{~min}$. Optical density (absorbance) was measured at $510 \mathrm{~nm}$ for each well using a microplate reader. TG $(\mathrm{mmol} / \mathrm{L})=\left(\mathrm{OD}_{\mathrm{EG}^{-}} \mathrm{OD}_{\mathrm{BG}}\right) /\left(\mathrm{OD}_{\mathrm{SG}^{-}}\right.$ $\left.\mathrm{OD}_{\mathrm{BG}}\right)$ * calibrator concentration $(\mathrm{mmol} / \mathrm{L})$.

\section{Quantitative real-time PCR}

Total RNA was extracted from harvested cells using TRIzol reagent (TaKaRa, Dalian, China), according to the manufacturer's instructions. RNA concentration was measured using a NanoDrop 2000 (Thermo, Waltham, MA, USA). cDNA was synthesized using the TaKaRa PrimeScript RT reagent kit with gDNA Eraser. Quantitative PCR was conducted using TaKaRa SYGB Premix EX Taq (Tli RNaseH Plus, CA). For miRNA analysis, the One Step PrimeScript miRNA cDNA Synthesis Kit (TaKaRa) and the SYBR PrimeScriptTM miRNA RTPCR Kit (TaKaRa) were used. Gene expression was normalized to $\beta$-actin, and the U6 small RNA was used as the internal reference for miRNA measurements. In real-time quantitative PCR, every reaction was performed in triplicate. Levels were calculated using the relative quantification $\left(2^{-\Delta \Delta \mathrm{Ct}}\right)$ method [61]. All primers are listed in Supplementary Table S6.

\section{Western blotting}

Cultured cells were washed two times with PBS, digested with $0.25 \%$ trypsin and then centrifuged at $1000 \mathrm{rpm}$ for $15 \mathrm{~min}$. The cells were homogenized in radioimmunoprecipitation assay (PIPA) lysis buffer (Beyotime, Shanghai, China) with phenylmethane sulfonyl fluoride (PMSF, Beyotime, Shanghai, China). Total protein was extracted from the supernatants after centrifugation at 12,000 rpm for $10 \mathrm{~min}$. Protein concentration was determined using a BCA Protein Assay Reagent Kit (Beyotime, Shanghai, China). A protein sample of $50 \mu \mathrm{g}$ was separated by SDS-PAGE, transferred to polyvinylidene fluoride (PVDF) membranes (Beyotime, Shanghai, China), and sealed overnight in $5 \%$ sealant. The membranes were washed three times for $10 \mathrm{~min}$ with $10 \times \mathrm{TBST}$, blocked with DifcoTM skim milk for $1 \mathrm{~h}$ at room temperature, and then incubated at $4{ }^{\circ} \mathrm{C}$ overnight with the following rabbit primary antibodies: anti-ACSL4 polyclonal antibody (1:1000, ABclonal, Wuhan, China), anti-Sirt1 polyclonal antibody (1:1000, ABclonal, Wuhan, China), anti-ATGL polyclonal antibody (1:1000, ABclonal, Wuhan, China), anti-aP2 polyclonal antibody (1: 1000, ABclonal, Wuhan, China), anti-SREBP-1c polyclonal antibody (1:1000, ABclonal, Wuhan, China), anti-PPAR $\gamma$ polyclonal antibody (1:1000, ABclonal, Wuhan, China), and antiGAPDH polyclonal antibody (1:1000, ABclonal, Wuhan, China). After three washes with $10 \times \mathrm{TBST}$, the second antibody (IgG 1:5000, ABclonal, Wuhan, China), conjugated with horseradish peroxidase, was added and the reaction was incubated at $37^{\circ} \mathrm{C}$ for $1 \mathrm{~h}$. Binding was detected using an ECL chemiluminescence kit (Beyotime, Shanghai, China). A gel imaging instrument (Vilber Lourmat fusion FX 7 Spectra, France) was used to scan the immunoblots, and an image analysis application (FUSIONCAPT, France) was used to determine the relative density of each band. The results are presented as the ratios of the optical densities of targeted proteins to those of GAPDH.

\section{Statistical analysis}

Statistical analyses were performed using SAS 9.4. All experiments were conducted in triplicate, and results are presented as means \pm SE. Multiple comparisons were assessed with a one-way analysis of variance followed by Dunnett's tests. $P$-values $<0.05$ were considered to be statistically significant.

\section{Supplementary information}

Supplementary information accompanies this paper at https://doi.org/10. 1186/s12863-020-0836-7.

Additional file 1: Supplementary Figure S1. Identification of porcine primary intramuscular preadipocytes. (A-B) Morphology of primary intramuscular preadipocytes observed under an inverted microscope $(x$ 
100) after cultivation for (A) 2 days and (B) 8 days. (C-F) Morphological changes and lipid accumulation in intramuscular adipocytes observed by Oil Red O staining ( $\times$ 400). Cells were collected at (C) 2, (D) 4, (E) 6, and (F) 8 days after induction of differentiation.

Additional file 2: Supplementary Table S1. miR-34a target genes predicted using TargetScan. Supplementary Table S2. miR-34a target genes predicted using miRDB. Supplementary Table S3. miR-34a target genes predicted both by TargetScan and miRDB. Supplementary Table

S4. The sequences of small interfering RNAs (siRNAs) specifically targeting ACSL4. Supplementary Table S5. Primers used for luciferase reporter assay. Supplementary Table S6. Primers used for real-time quantitative PCR.

\section{Abbreviations}

IMF: intramuscular fat; ACSL4: acyl-CoA synthetase long chain family member 4; CASR: calcium-sensing receptor; GO: Gene Ontology; KEGG: Kyoto Encyclopaedia of Genes and Genomes; LD: Iongissimus dorsi muscle; CoA: coenzyme-A; ACSL: long-chain acyl-CoA synthetase; PBS: phosphatebuffered saline

\section{Acknowledgements}

Not applicable.

\section{Authors' contributions}

$H T, W W$, and $X L$ conceived this study and wrote the manuscript. $X L, N D$, and $J T$ were responsible for animal care, sample preparation, and the performance of experiments. QZ and SZ performed the data processing. All authors reviewed and approved the final manuscript.

\section{Funding}

This work was supported by the National Natural Science Foundation of China (awards 31702093 and 31572361; for travel and sampling costs, and to support data analysis and interpretation), the China Postdoctoral Science Foundation (2019 M652449), and the Shandong "Double Tops" Program (award SYL2017YSTD12; for data analysis and interpretation, and for manuscript preparation)

\section{Availability of data and materials}

All data generated or analyzed during this study are included in this published article and its supplementary files.

\section{Ethics approval and consent to participate}

Animal experiments were carried out in accordance with the Guidelines for Experimental Animals established by the Ministry of Science and Technology (Beijing, China). The protocol was approved by the Institutional Animal Care and Use Ethics Committee of Shandong Agricultural University.

\section{Consent for publication}

Not applicable.

\section{Competing interests}

The authors declare that they have no competing interests.

Received: 27 May 2019 Accepted: 4 March 2020

Published online: 14 March 2020

\section{References}

1. Fernandez X, Monin G, Talmant A, Mourot J, Lebret B. Influence of intramuscular fat content on the quality of pig meat - 1. Composition of the lipid fraction and sensory characteristics of $\mathrm{m}$. longissimus lumborum. Meat Sci. 1999:53(1):59-65.

2. Hocquette JF, Gondret F, Baeza E, Medale F, Jurie C, Pethick DW. Intramuscular fat content in meat-producing animals: development, genetic and nutritional control, and identification of putative markers. Animal. 2010;4(2):303-19.

3. Davoli R, Luise D, Mingazzini V, Zambonelli P, Braglia S, Serra A, Russo V. Genome-wide study on intramuscular fat in Italian large white pig breed using the PorcineSNP60 BeadChip. J Anim Breed Genet. 2016;133(4):277-82.

4. Puig-Oliveras A, Revilla M, Castello A, Fernandez Al, Folch JM, Ballester M. Expression-based GWAS identifies variants, gene interactions and key regulators affecting intramuscular fatty acid content and composition in porcine meat. Sci Rep. 2016:6:31803.

5. Munoz M, Rodriguez MC, Alves E, Folch JM, Ibanez-Escriche N, Silio L, Fernandez Al. Genome-wide analysis of porcine backfat and intramuscular fat fatty acid composition using high-density genotyping and expression data. BMC Genomics. 2013;14:845.

6. Wang Y, Ning C, Wang C, Guo J, Wang J, Wu Y. Genome-wide association study for intramuscular fat content in Chinese Lulai black pigs. AsianAustralas J Anim Sci. 2019;32(5):607-13.

7. Ros-Freixedes R, Gol S, Pena RN, Tor M, Ibanez-Escriche N, Dekkers JC, Estany J. Genome-wide association study singles out SCD and LEPR as the two Main loci influencing intramuscular fat content and fatty acid composition in Duroc pigs. PLoS One. 2016:11(3):e152496.

8. Won S, Jung J, Park E, Kim H. Identification of genes related to intramuscular fat content of pigs using genome-wide association study. Asian-Australas J Anim Sci. 2018;31(2):157-62.

9. Li W, Yang Y, Liu Y, Liu S, Li X, Wang Y, Zhang Y, Tang H, Zhou R, Li K Integrated analysis of mRNA and miRNA expression profiles in livers of Yimeng black pigs with extreme phenotypes for backfat thickness. Oncotarget. 2017;8(70):114787-800,

10. Bartel DP. MicroRNAs: genomics, biogenesis, mechanism, and function. Cell. 2004;116(2):281-97.

11. He L, Hannon GJ. MicroRNAs: small RNAs with a big role in gene regulation Nat Rev Genet. 2004;5(7):522-31.

12. Tufekci KU, Meuwissen RL, Genc S. The role of microRNAs in biological processes. Methods Mol Biol. 2014;1107:15-31.

13. Bhaskaran M, Mohan M. MicroRNAs: history, biogenesis, and their evolving role in animal development and disease. Vet Pathol. 2014:51(4):759-74.

14. Novak J, Bienertova-Vasku J, Kara T, Novak M. MicroRNAs involved in the lipid metabolism and their possible implications for atherosclerosis development and treatment. Mediat Inflamm. 2014;2014:275867.

15. Di Leva G, Briskin D, Croce CM. MicroRNA in cancer: new hopes for antineoplastic chemotherapy. Ups J Med Sci. 2012;117(2):202-16.

16. Ning X, Liu S, Qiu Y, Li G, Li Y, Li M, Yang G. Expression Profiles and Biological Roles of miR-196a in Swine. Genes (Basel). 2016;7(2):5.

17. Yang W, Tang K, Wang Y, Zan L. MiR-27a-5p increases steer fat deposition partly by targeting calcium-sensing receptor (CASR). Sci Rep. 2018;8(1):3012.

18. Wang J, Guan X, Guo F, Zhou J, Chang A, Sun B, Cai Y, Ma Z, Dai C, Li X, et al. miR-30e reciprocally regulates the differentiation of adipocytes and osteoblasts by directly targeting low-density lipoprotein receptor-related protein 6. Cell Death Dis. 2013;4:e845.

19. Lee EK, Lee MJ, Abdelmohsen K, Kim W, Kim MM, Srikantan S, Martindale JL Hutchison ER, Kim HH, Marasa BS, et al. miR-130 suppresses adipogenesis by inhibiting peroxisome proliferator-activated receptor gamma expression. Mol Cell Biol. 2011;31(4):626-38.

20. Chen Y, Siegel F, Kipschull S, Haas B, Frohlich H, Meister G, Pfeifer A. miR155 regulates differentiation of brown and beige adipocytes via a bistable circuit. Nat Commun. 2013;4:1769.

21. Wen D, Peng Y, Lin F, Singh RK, Mahato RI. Micellar delivery of miR-34a modulator Rubone and paclitaxel in resistant prostate Cancer. Cancer Res. 2017;77(12):3244-54

22. Ito $Y$, Inoue $A$, Seers $T$, Hato $Y$, Igarashi A, Toyama T, Taganov KD, Boldin MP, Asahara $\mathrm{H}$. Identification of targets of tumor suppressor microRNA-34a using a reporter library system. Proc Natl Acad Sci U S A 2017:114(15):3927-32.

23. Shi Y, Liu C, Liu X, Tang DG, Wang J. The microRNA miR-34a inhibits nonsmall cell lung cancer (NSCLC) growth and the CD44hi stem-like NSCLC cells. PLoS One. 2014;9(3):e90022.

24. Adams BD, Parsons C, Slack FJ. The tumor-suppressive and potential therapeutic functions of miR-34a in epithelial carcinomas. Expert Opin Ther Targets. 2016;20(6):737-53.

25. Saito $Y$, Nakaoka T, Saito H. microRNA-34a as a therapeutic agent against human Cancer. J Clin Med. 2015;4(11):1951-9.

26. Li L. Regulatory mechanisms and clinical perspectives of miR-34a in cancer. J Cancer Res Ther. 2014;10(4):805-10.

27. Li XJ, Ren ZJ, Tang JH. MicroRNA-34a: a potential therapeutic target in human cancer. Cell Death Dis. 2014;5:e1327.

28. Maroni P, Puglisi R, Mattia G, Care A, Matteucci E, Bendinelli P, Desiderio MA. In bone metastasis miR-34a-5p absence inversely correlates with met expression, while met oncogene is unaffected by miR-34a-5p in non-metastatic and metastatic breast carcinomas. Carcinogenesis. 2017;38(5):492-503. 
29. Xiang ZL, Zhao XM, Zhang L, Yang P, Fan J, Tang ZY, Zeng ZC. MicroRNA-34a expression levels in serum and intratumoral tissue can predict bone metastasis in patients with hepatocellular carcinoma. Oncotarget. 2016;7(52):87246-56.

30. Ghandadi M, Sahebkar A. MicroRNA-34a and its target genes: key factors in cancer multidrug resistance. Curr Pharm Des. 2016;22(7):933-9.

31. Rapti SM, Kontos CK, Christodoulou S, Papadopoulos IN, Scorilas A. miR-34a overexpression predicts poor prognostic outcome in colorectal adenocarcinoma, independently of clinicopathological factors with established prognostic value. Clin Biochem. 2017;50(16-17):918-24.

32. Imani S, Zhang $X$, Hosseinifard H, Fu S, Fu J. The diagnostic role of microRNA-34a in breast cancer: a systematic review and meta-analysis. Oncotarget. 2017:8(14):23177-87.

33. Chen AH, Qin YE, Tang WF, Tao J, Song HM, Zuo M. MiR-34a and miR-206 act as novel prognostic and therapy biomarkers in cervical cancer. Cancer Cell Int. 2017;17:63.

34. Zhao X, Sun Y, Ding Y, Zhang J, Li K. miR-34a inhibitor may effectively protect against Sevoflurane-induced hippocampal apoptosis through the Wnt/betacatenin pathway by targeting Wnt1. Yonsei Med J. 2018;59(10):1205-13.

35. Torres JL, Novo-Veleiro I, Manzanedo L, Alvela-Suarez L, Macias R, Laso FJ, Marcos M. Role of microRNAs in alcohol-induced liver disorders and nonalcoholic fatty liver disease. World J Gastroenterol. 2018;24(36):4104-18.

36. Tao YF, Qiang J, Bao JW, Chen DJ, Yin GJ, Xu P, Zhu HJ. Changes in physiological parameters, lipid metabolism, and expression of MicroRNAs in genetically improved farmed Tilapia (Oreochromis niloticus) with fatty liver induced by a high-fat diet. Front Physiol. 2018;9:1521.

37. Xu Y, Du J, Zhang P, Zhao X, Li Q, Jiang A, Jiang D, Tang G, Jiang Y, Wang J, et al. MicroRNA-125a-5p Mediates 3T3-L1 Preadipocyte Proliferation and Differentiation. Molecules. 2018;23(2):317.

38. Kim C, Lee H, Cho YM, Kwon OJ, Kim W, Lee EK. TNFalpha-induced miR-130 resulted in adipocyte dysfunction during obesity-related inflammation. FEBS Lett. 2013;S0014-5793(13):00775-8.

39. Mashek DG, Li LO, Coleman RA. Long-chain acyl-CoA synthetases and fatty acid channeling. Future Lipidol. 2007;2(4):465-76.

40. Li LO, Mashek DG, An J, Doughman SD, Newgard CB, Coleman RA. Overexpression of rat long chain acyl-coa synthetase 1 alters fatty acid metabolism in rat primary hepatocytes. J Biol Chem. 2006;281(48):37246-55.

41. Xu X, Gopalacharyulu P, Seppanen-Laakso T, Ruskeepaa AL, Aye CC, Carson BP, Mora S, Oresic M, Teleman AA. Insulin signaling regulates fatty acid catabolism at the level of CoA activation. PLoS Genet. 2012;8(1):e1002478.

42. Duarte A, Poderoso C, Cooke M, Soria G, Cornejo MF, Gottifredi V, Podesta EJ. Mitochondrial fusion is essential for steroid biosynthesis. PLoS One. 2012; 7(9):e45829.

43. Maloberti PM, Duarte AB, Orlando UD, Pasqualini ME, Solano AR, Lopez-Otin C, Podesta EJ. Functional interaction between acyl-CoA synthetase 4, lipooxygenases and cyclooxygenase-2 in the aggressive phenotype of breast cancer cells. PLoS One. 2010;5(11):e15540.

44. Kuwata H, Hara S. Inhibition of long-chain acyl-CoA synthetase 4 facilitates production of 5, 11-dihydroxyeicosatetraenoic acid via the cyclooxygenase2 pathway. Biochem Biophys Res Commun. 2015;465(3):528-33.

45. Kang MJ, Fujino T, Sasano H, Minekura H, Yabuki N, Nagura H, lijima H, Yamamoto $\Pi$. A novel arachidonate-preferring acyl-CoA synthetase is present in steroidogenic cells of the rat adrenal, ovary, and testis. Proc Natl Acad Sci U S A. 1997;94(7):2880-4.

46. Klett EL, Chen S, Edin ML, Li LO, Ilkayeva O, Zeldin DC, Newgard CB, Coleman RA. Diminished acyl-CoA synthetase isoform 4 activity in INS 832/13 cells reduces cellular epoxyeicosatrienoic acid levels and results in impaired glucose-stimulated insulin secretion. J Biol Chem. 2013;288(30):21618-29.

47. Mercade A, Estelle J, Perez-Enciso M, Varona L, Silio L, Noguera JL, Sanchez A, Folch JM. Characterization of the porcine acyl-CoA synthetase long-chain 4 gene and its association with growth and meat quality traits. Anim Genet. 2006;37(3):219-24

48. Rusc A, Sieczkowska H, Krzecio E, Antosik K, Zybert A, Kocwin-Podsiadla M, Kaminski S. The association between acyl-CoA synthetase (ACSL4) polymorphism and intramuscular fat content in (landrace $\mathrm{x}$ Yorkshire) $\mathrm{x}$ Duroc pigs. Meat Sci. 2011;89(4):440-3.

49. Chen JN, Jiang YZ, Cen WM, Xing SH, Zhu L, Tang GQ, Li MZ, Jiang AA, Lou PE, Wen AX, et al. Distribution of H-FABP and ACSL4 gene polymorphisms and their associations with intramuscular fat content and backfat thickness in different pig populations. Genet Mol Res. 2014;13(3):6759-72.

50. Gregoire FM, Smas CM, Sul HS. Understanding adipocyte differentiation. Physiol Rev. 1998;78(3):783-809.
51. Morrison RF, Farmer SR. Hormonal signaling and transcriptional control of adipocyte differentiation. J Nutr. 2000;130(12):3116S-21S

52. Lasa A, Churruca I, Eseberri I, Andres-Lacueva C, Portillo MP. Delipidating effect of resveratrol metabolites in 3T3-L1 adipocytes. Mol Nutr Food Res. 2012:56(10):1559-68

53. Sun YM, Qin J, Liu SG, Cai R, Chen XC, Wang XM, Pang WJ. PDGFRalpha Regulated by miR-34a and FoxO1 Promotes Adipogenesis in Porcine Intramuscular Preadipocytes through Erk Signaling Pathway. Int J Mol Sci. 2017:18(11):2424.

54. Wen F, An C, Wu X, Yang Y, Xu J, Liu Y, Wang C, Nie L, Fang H, Yang Z. MiR34 a regulates mitochondrial content and fat ectopic deposition induced by resistin through the AMPK/PPARalpha pathway in HepG2 cells. Int J Biochem Cell Biol. 2018;94:133-45.

55. Piletič K, Kunej T. Minimal standards for reporting microRNA: target interactions. Omics. 2017:21(4):197-206.

56. Agarwal V, Bell GW, Nam J, Bartel DP. Predicting effective microRNA target sites in mammalian mRNAs. eLife. 2015;4:e05005.

57. Liu W, Wang X. Prediction of functional microRNA targets by integrative modeling of microRNA binding and target expression data. Genome Biol. 2019;20(1):18.

58. Rehmsmeier M, Steffen $P$, Hochsmann M, Giegerich R. Fast and effective prediction of microRNA/target duplexes. RNA. 2004;10:1507-17.

59. $Y u$ G, Wang LG, Han Y, He QY. clusterProfiler: an R package for comparing biological themes among gene clusters. OMICS. 2012;16(5):284-7.

60. Wilkinson L. ggplot2: elegant graphics for data analysis by H. WICKHAM. Biometrics. 2011:67(2):678-9.

61. Livak KJ, Schmittgen TD. Analysis of relative gene expression data using real-time quantitative PCR and the $2(-$ Delta Delta $C(T))$ method. Methods. 2001;25(4):402-8.

\section{Publisher's Note}

Springer Nature remains neutral with regard to jurisdictional claims in published maps and institutional affiliations.
Ready to submit your research? Choose BMC and benefit from:

- fast, convenient online submission

- thorough peer review by experienced researchers in your field

- rapid publication on acceptance

- support for research data, including large and complex data types

- gold Open Access which fosters wider collaboration and increased citations

- maximum visibility for your research: over $100 \mathrm{M}$ website views per year

At $\mathrm{BMC}$, research is always in progress.

Learn more biomedcentral.com/submissions 